\title{
Long-Term Survival after Stroke in 1.4 Million Japanese Population: Shiga Stroke and Heart Attack Registry
}

\author{
Naoyuki Takashima, ${ }^{\mathrm{a}, \mathrm{b}}$ Hisatomi Arima, ${ }^{\mathrm{c}}$ Yoshikuni Kita, ${ }^{\mathrm{a}, \mathrm{d}}$ Takako Fujii, ${ }^{\mathrm{c}, \mathrm{e}}$ Sachiko Tanaka-Mizuno, \\ Satoshi Shitara, ${ }^{\mathrm{e}}$ Akihiro Kitamura, ${ }^{\mathrm{g}}$ Yoshihisa Sugimoto, ${ }^{\mathrm{h}}$ Makoto Urushitani, ${ }^{\mathrm{g}}$ Katsuyuki Miura, ${ }^{\mathrm{a}, \mathrm{i}}$ \\ Kazuhiko Nozakie,i \\ aDepartment of Public Health, Shiga University of Medical Science, Otsu, Japan \\ ${ }^{b}$ Department of Public Health, Kindai University Faculty of Medicine, Osaka-Sayama, Japan \\ 'Department of Preventive Medicine and Public Health, Fukuoka University Faculty of Medicine, Fukuoka, Japan

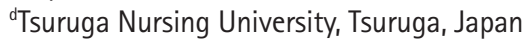 \\ 'Department of Neurosurgery, Shiga University of Medical Science, Otsu, Japan \\ fDepartment of Medical Statistics, Shiga University of Medical Science, Otsu, Japan \\ ${ }^{9}$ Department of Neurology, Shiga University of Medical Science, Otsu, Japan \\ hDepartment of Medical Informatics and Biomedical Engineering, Shiga University of Medical Science, Otsu, Japan \\ 'Center for Epidemiologic Research in Asia, Shiga University of Medical Science, Otsu, Japan
}

Background and Purpose Although numerous measures for stroke exist, stroke remains one of the leading causes of death in Japan. In this study, we aimed to determine the long-term survival rate after first-ever stroke using data from a large-scale population-based stroke registry study in Japan. Methods Part of the Shiga Stroke and Heart Attack Registry, the Shiga Stroke Registry is an ongoing population-based registry study of stroke, which covers approximately 1.4 million residents of Shiga Prefecture in Japan. A total 1,880 patients with non-fatal first-ever stroke (among 29-day survivors after stroke onset) registered in 2011 were followed up until December 2016. Five-year cumulative survival rates were estimated using the Kaplan-Meier method, according to subtype of the index stroke. Cox proportional hazards models were used to assess predictors of subsequent allcause death.

Results During an average 4.3-year follow-up period, 677 patients died. The 5-year cumulative survival rate after non-fatal first-ever stroke was $65.9 \%$. Heterogeneity was present in 5-year cumulative survival according to stroke subtype: lacunar infarction, 75.1\%; large-artery infarction, 61.5\%; cardioembolic infarction, 44.9\%; intracerebral hemorrhage, 69.1\%; and subarachnoid hemorrhage, 77.9\%. Age, male sex, Japan Coma Scale score on admission, and modified Rankin Scale score before stroke onset were associated with increased mortality during the chronic phase of ischemic and hemorrhagic stroke.

Conclusions In this study conducted in a real-world setting of Japan, the 5-year survival rate after non-fatal first-ever stroke remained low, particularly among patients with cardioembolic infarction and large-artery infarction in the present population-based stroke registry.
Correspondence: Naoyuki Takashima Department of Public Health, Kindai University Faculty of Medicine, OsakaSayama 377-2, Osaka 589-8511, Japan Tel: +81-72-366-0221 (ext.3271)

Fax: +81-72-367-8262

E-mail: n.takashima@med.kindai.ac.jp https://orcid.org/0000-0002-95936797

Received: Janaury 29, 2020

Revised: June 29, 2020

Accepted: July 29, 2020

Keywords Stroke; Epidemiology; Population surveillance; Survival rate

Copyright ( ) 2020 Korean Stroke Society

This is an Open Access article distributed under the terms of the Creative Commons Attribution Non-Commercial License (http://creativecommons.org/licenses/by-nc/4.0/) which permits unrestricted non-commercial use, distribution, and reproduction in any medium, provided the original work is properly cited. 


\section{Introduction}

During the past five decades, age-standardized stroke mortality has decreased in Japan. ${ }^{1}$ However, in Japan, stroke is still one of the leading causes of death ${ }^{2}$ and disability. ${ }^{3}$ In all regions of the world, the age-adjusted stroke mortality rate has rapidly decreased whereas the incidence rate has less steep decreased in high-income Asian pacific or Southeast Asian countries or increased in China. ${ }^{4}$ The rapid decrease in age-adjusted mortality rates and less steep decrease or increase in age-adjusted incidence rates have led to an increased number of stroke survivors. ${ }^{4}$ Using data from the Shiga Stroke registry study, we previously reported that case fatality rates were lower than those of previous studies from Japan and other countries, ${ }^{5}$ which might also suggest increase in number of stroke survivors during the past few decades. The excess risk of death has been reported to be highest during the acute phase but persists during the several years following stroke. ${ }^{6-8}$ However, there is limited evidence of long-term mortality among patients who experience non-fatal stroke in Japan, which is the most aged society and the first nation to face a super-aging society in the world. ${ }^{9}$

As a part of the Shiga Stroke and Heart Attack Registry, the Shiga Stroke Registry is one of the largest ongoing populationbased registry study of stroke in Japan. We previously reported the incidence rate ${ }^{5}$ and both acute and 2-year survival rates ${ }^{10}$ of stroke, using data from this study. We aimed to investigate the current cumulative and relative 5-year survival rates after non-fatal first-ever stroke, using a large-scale comprehensive population-based stroke registry in Shiga Prefecture, Japan.

\section{Methods}

\section{Study design}

The design of the Shiga Stroke Registry has been described elsewhere in detail. ${ }^{5,10}$ The Shiga Stroke Registry is a populationbased registry study designed to build a complete information system regarding acute ischemic and non-traumatic hemorrhagic stroke management in Shiga Prefecture, Japan. Shiga Prefecture is located in the central part of Honshu Island. According to the 2011 census, the population of Shiga Prefecture was $1,400,745$ residents (689,859 men and 710,866 women).

Diagnosis of stroke was defined as a sudden onset of focal neurological deficits persisting for more than 24 hours, according to the Monitoring Trends and Determinants in Cardiovascular Disease (WHO-MONICA) Project. ${ }^{11} \mathrm{~A}$ total 2,176 cases of first-ever stroke, with onset dates ranging from 1 January to 31 December in 2011, were followed up until December 2016. A patient with non-fatal stroke was defined as a person who ex- perienced stroke and was alive at 29 days after onset of the index stroke. We excluded 296 patients with fatal stroke who died within 28 days. A total 1,880 patients with non-fatal firstever stroke were included in the present analysis. A sensitivity analysis using 2,176 fatal/non-fatal first-ever stroke patients was also conducted. The present study was approved by the Institutional Review Board of Shiga University of Medical Science. Written informed consent by the patients was waived due to a retrospective nature of our study.

\section{Definition of index stroke and its subtypes}

Stroke was classified as ischemic or hemorrhagic stroke. Ischemic stroke was further divided into four clinical categories: lacunar infarction, large-artery infarction, cardioembolic infarction, or undetermined type, based on the criteria for the type of stroke of the Trial of ORG 10172 in Acute Stroke Treatment (TOAST) study. ${ }^{12}$ Hemorrhagic stroke was further divided into two clinical categories: intracerebral hemorrhage and subarachnoid hemorrhage.

\section{Clinical features and medical histories}

Information on clinical features, including transient ischemic attack (TIA), atrial fibrillation, myocardial infarction, hypertension medication, diabetes, dyslipidemia, modified Rankin Scale (mRS) score ${ }^{13}$ before onset, and smoking and drinking status at baseline, was obtained from the medical records of all patients with stroke. Atrial fibrillation was defined as a history of atrial fibrillation and/or a clinical diagnosis based on electrocardiogram (ECG) and/or ECG monitoring during hospitalization. The Japan Coma Scale (JCS) ${ }^{14}$ score is a 10 -grade scale that is widely used in Japan to assess consciousness (JCS 0, alert; JCS 1-3, possible eye-opening, not lucid; JCS 10-30, possible eyeopening upon stimulation; JCS 100-300, no eye-opening, coma) (Supplementary Table 1). Hypertension medication was defined as the use of antihypertensive medication before onset of the index stroke. Diabetes was defined as casual blood glucose $\geq 11.1 \mathrm{mmol} / \mathrm{L}$, use of antidiabetic medication, and/or a history of diabetes. Dyslipidemia was defined as total cholesterol $\geq 5.69 \mathrm{mmol} / \mathrm{L}$, low density lipoprotein cholesterol $\geq 4.14$ $\mathrm{mmol} / \mathrm{L}$, taking medication for dyslipidemia, and/or a history of dyslipidemia. Smoking and drinking habits were categorized as none, past, and current.

\section{Follow-up survey and outcome}

All patients with first-ever stroke were followed up until December 2016, using death certificate information. Information from the death certificates of all deceased individuals was collected, with the permission of the Japanese Ministry of Health, 
Table 1. Characteristics of non-fatal first-ever stroke patients survived acute phase by type of index stroke in the Shiga Stroke Registry

\begin{tabular}{|c|c|c|c|}
\hline Characteristic & $\begin{array}{l}\text { Ischemic } \\
\text { stroke }\end{array}$ & $\begin{array}{l}\text { Hemorrhagic } \\
\text { stroke }\end{array}$ & Total stroke \\
\hline Total no. & 1,296 & 583 & 1,880 \\
\hline Men & $743(57.3)$ & $260(44.6)$ & $1,003(53.4)$ \\
\hline Age (yr) & $74.1 \pm 13.2$ & $68.2 \pm 15.1$ & $72.3 \pm 14.1$ \\
\hline \multicolumn{4}{|l|}{ Past history } \\
\hline \multicolumn{4}{|c|}{ Transient ischemic attacks } \\
\hline Yes & $71(5.5)$ & $8(1.4)$ & $79(4.2)$ \\
\hline No & $1,222(94.3)$ & 572 (98.1) & $1,795(95.5)$ \\
\hline Unknown & $3(0.2)$ & $3(0.5)$ & $6(0.3)$ \\
\hline \multicolumn{4}{|c|}{ Atrial fibrillation } \\
\hline Yes & $286(22.1)$ & $38(6.5)$ & $324(17.2)$ \\
\hline No & $1,004(77.5)$ & $540(92.6)$ & $1,545(82.2)$ \\
\hline Unknown & $6(0.5)$ & $5(0.9)$ & $11(0.6)$ \\
\hline \multicolumn{4}{|c|}{ Myocardial infarction } \\
\hline Yes & $79(6.1)$ & $27(4.6)$ & $107(5.7)$ \\
\hline No & 1,215 (93.8) & 554 (95) & $1,769(94.1)$ \\
\hline Unknown & $2(0.2)$ & $2(0.3)$ & $4(0.2)$ \\
\hline \multicolumn{4}{|c|}{ Hypertension medication } \\
\hline Yes & $656(50.6)$ & $224(38.4)$ & $880(46.8)$ \\
\hline No & 635 (49) & 355 (60.9) & $991(52.7)$ \\
\hline Unknown & $5(0.4)$ & $4(0.7)$ & $9(0.5)$ \\
\hline \multicolumn{4}{|l|}{ Diabetes } \\
\hline Yes & $360(27.8)$ & $114(19.6)$ & $474(25.2)$ \\
\hline No & $930(71.8)$ & 466 (79.9) & $1,397(74.3)$ \\
\hline Unknown & $6(0.5)$ & $3(0.5)$ & $9(0.5)$ \\
\hline \multicolumn{4}{|l|}{ Dyslipidemia } \\
\hline Yes & $510(39.4)$ & 158 (27.1) & $668(35.5)$ \\
\hline No & 748 (57.7) & 377 (64.7) & $1,126(59.9)$ \\
\hline Unknown & $38(2.9)$ & $48(8.2)$ & $86(4.6)$ \\
\hline \multicolumn{4}{|c|}{$\begin{array}{l}\text { Modified Rankin Scale be- } \\
\text { fore on set }\end{array}$} \\
\hline 0 & 907 (70.0) & 450 (77.2) & $1,358(72.2)$ \\
\hline 1 & 133 (10.3) & $48(8.2)$ & 181 (9.6) \\
\hline 2 & $57(4.4)$ & $35(6.0)$ & $92(4.9)$ \\
\hline 3 & $86(6.6)$ & $17(2.9)$ & $103(5.5)$ \\
\hline 4 & $81(6.3)$ & $17(2.9)$ & $98(5.2)$ \\
\hline 5 & $26(2.0)$ & $3(0.5)$ & $29(1.5)$ \\
\hline Unknown & $6(0.5)$ & $13(2.2)$ & $19(1.0)$ \\
\hline \multicolumn{4}{|c|}{$\begin{array}{l}\text { Japan Coma Scale on ad- } \\
\text { mission }\end{array}$} \\
\hline 0 & $810(62.5)$ & $206(35.3)$ & $1,017(54.1)$ \\
\hline 1 & 114 (8.8) & 70 (12) & 184 (9.8) \\
\hline 2 & $77(5.9)$ & $32(5.5)$ & 109 (5.8) \\
\hline 3 & $122(9.4)$ & $55(9.4)$ & 177 (9.4) \\
\hline 10 & $64(4.9)$ & $102(17.5)$ & 166 (8.8) \\
\hline
\end{tabular}

Table 1. Continued

\begin{tabular}{|c|c|c|c|}
\hline Characteristic & $\begin{array}{l}\text { Ischemic } \\
\text { stroke }\end{array}$ & $\begin{array}{l}\text { Hemorrhagic } \\
\text { stroke }\end{array}$ & Total stroke \\
\hline 20 & $22(1.7)$ & $18(3.1)$ & $40(2.1)$ \\
\hline 30 & $20(1.5)$ & $14(2.4)$ & $34(1.8)$ \\
\hline 100 & $18(1.4)$ & $22(3.8)$ & $40(2.1)$ \\
\hline 200 & $35(2.7)$ & $45(7.7)$ & $80(4.3)$ \\
\hline 300 & $11(0.8)$ & $17(2.9)$ & $28(1.5)$ \\
\hline Unknown & $3(0.2)$ & $2(0.3)$ & $5(0.3)$ \\
\hline \multicolumn{4}{|l|}{ Smoking } \\
\hline Nonsmoker & $734(56.6)$ & $352(60.4)$ & $1,087(57.8)$ \\
\hline Past smoker & $152(11.7)$ & $57(9.8)$ & $209(11.1)$ \\
\hline Current smoker & 309 (23.8) & $129(22.1)$ & $438(23.3)$ \\
\hline Unknown & $101(7.8)$ & $45(7.7)$ & $146(7.8)$ \\
\hline \multicolumn{4}{|l|}{ Drinking } \\
\hline Nondrinker & $642(49.5)$ & $296(50.8)$ & $938(49.9)$ \\
\hline Past drinker & $21(1.6)$ & $7(1.2)$ & $28(1.5)$ \\
\hline Current drinker & $468(36.1)$ & $213(36.5)$ & $682(36.3)$ \\
\hline Unknown & 165 (12.7) & $67(11.5)$ & $232(12.3)$ \\
\hline \multicolumn{4}{|l|}{ Index stroke subtype } \\
\hline Ischemic stroke & $1,296(100.0)$ & & $1,296(68.9)$ \\
\hline Lacunar infarction & $346(26.7)$ & & $346(18.4)$ \\
\hline Large artery infarction & 413 (31.9) & & $413(22.0)$ \\
\hline Cardioembolic infarction & $305(23.5)$ & & $305(16.2)$ \\
\hline $\begin{array}{l}\text { Ischemic stroke with oth- } \\
\text { er determined etiology }\end{array}$ & $38(2.9)$ & & $38(2.0)$ \\
\hline $\begin{array}{l}\text { Ischemic stroke with un- } \\
\text { determined etiology }\end{array}$ & 194 (15.0) & & 194 (10.3) \\
\hline Intracerebral hemorrhage & & $447(76.7)$ & $447(23.8)$ \\
\hline Subarachnoid hemorrhage & & $136(23.3)$ & $136(7.2)$ \\
\hline $\begin{array}{l}\text { Stroke, not specified as } \\
\text { hemorrhage or infarction }\end{array}$ & & & $1(0.1)$ \\
\hline
\end{tabular}

Values are presented as number (\%) or mean \pm standard deviation.

Labor and Welfare, and matched with all stroke cases. The main outcome of this study was all-cause death.

\section{Statistical analysis}

Cumulative survival rates of first-ever stroke cases at 5 years were estimated using Kaplan-Meier methods. Log-rank tests were used to compare survival rates across subtypes of stroke and ischemic stroke. Relative survival rate was defined as the ratio of observation survival rate during the follow-up period to the expected survival rate in the population matched by age, sex, and calendar year of the observational group. The expected survival rate was calculated using the Ederer I method ${ }^{15}$ and Japanese cohort survival tables. ${ }^{16}$ In addition, excess mortality risk was calculated, to compare the cumulative mortality rate with the expected mo- 
Table 2. Cumulative 5-year survival rate after non-fatal first-ever stroke by types of stroke and gender in the Shiga Stroke Registry

\begin{tabular}{|c|c|c|c|c|c|c|c|c|c|}
\hline \multirow[b]{2}{*}{ Variable } & \multicolumn{3}{|c|}{ Men } & \multicolumn{3}{|c|}{ Women } & \multicolumn{3}{|c|}{ Men and Women } \\
\hline & $\begin{array}{c}\text { Death/total } \\
\text { no. }\end{array}$ & $\begin{array}{c}\text { Survival rate } \\
(\%)\end{array}$ & $95 \% \mathrm{Cl}$ & $\begin{array}{c}\text { Death/total } \\
\text { no. }\end{array}$ & $\begin{array}{c}\text { Survival rate } \\
(\%)\end{array}$ & $95 \% \mathrm{Cl}$ & $\begin{array}{c}\text { Death/total } \\
\text { no. }\end{array}$ & $\begin{array}{c}\text { Survival rate } \\
(\%)\end{array}$ & $95 \% \mathrm{Cl}$ \\
\hline Total stroke & $319 / 1,003$ & 68.2 & $66.7-69.7$ & $322 / 877$ & 63.3 & $61.7-64.9$ & $641 / 1,880$ & 65.9 & $64.8-67.0$ \\
\hline Ischemic stroke & $247 / 743$ & 66.8 & $65.0-68.5$ & $226 / 553$ & 59.1 & $57.0-61.2$ & $473 / 1,296$ & 63.5 & $62.2-64.8$ \\
\hline Lacunar infarction & $50 / 208$ & 76.0 & $73.0-78.9$ & $36 / 138$ & 73.9 & $70.2-77.7$ & $86 / 346$ & 75.1 & $72.8-77.5$ \\
\hline Large artery infarction & $91 / 252$ & 63.9 & $60.9-66.9$ & $68 / 161$ & 57.8 & $53.9-61.7$ & $159 / 413$ & 61.5 & $59.1-63.9$ \\
\hline Cardioembolic infarction & $79 / 152$ & 48.0 & $44.0-52.1$ & $89 / 153$ & 41.8 & $37.8-45.8$ & $168 / 305$ & 44.9 & $42.1-47.8$ \\
\hline Other/undetermined ${ }^{*}$ & $27 / 131$ & 79.4 & $75.9-82.9$ & $33 / 101$ & 67.3 & $62.7-72.0$ & $60 / 232$ & 74.1 & $71.3-77.0$ \\
\hline Hemorrhagic stroke & $72 / 260$ & 72.3 & $69.5-75.1$ & $96 / 323$ & 70.3 & $67.7-72.8$ & $168 / 583$ & 71.2 & $69.3-73.1$ \\
\hline Intracerebral hemorrhage & $64 / 221$ & 71.0 & $68.0-74.1$ & $74 / 226$ & 67.3 & $64.1-70.4$ & $138 / 447$ & 69.1 & $66.9-71.3$ \\
\hline Subarachnoid hemorrhage & $8 / 39$ & 79.5 & $73.0-86.0$ & $22 / 97$ & 77.3 & $73.1-81.6$ & $30 / 136$ & 77.9 & $74.4-81.5$ \\
\hline
\end{tabular}

$\mathrm{Cl}$, confidence interval.

*Ischemic stroke with other determined etiology or ischemic stroke with undetermined etiology.

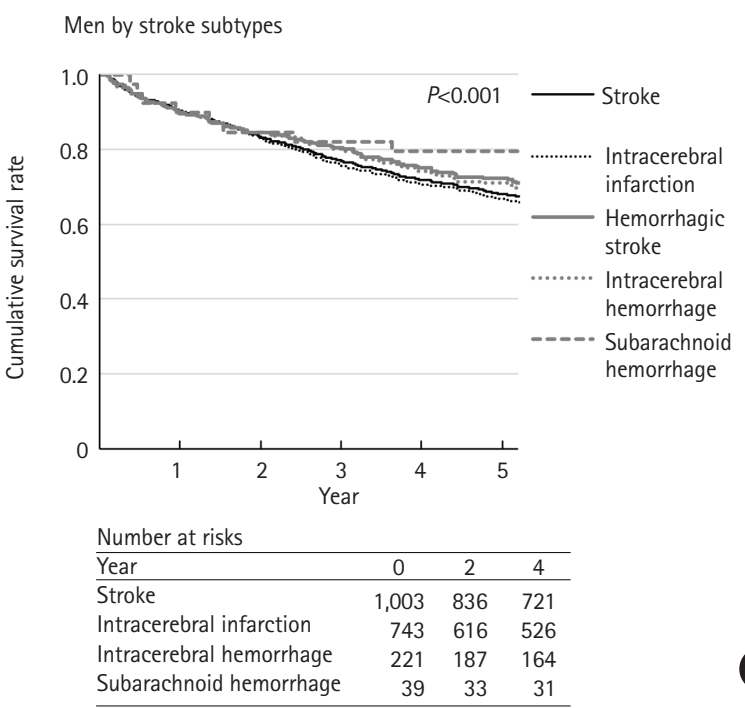

Women by stroke subtypes

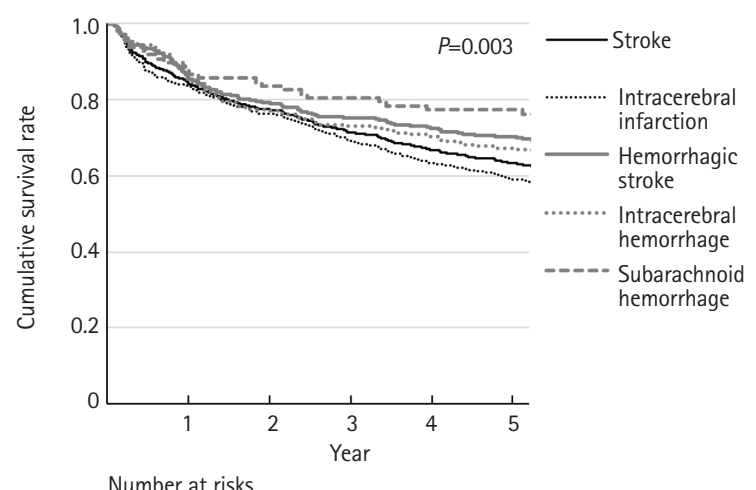

\begin{tabular}{lccc} 
Number at risks & \multicolumn{1}{c}{ ( } & \multicolumn{1}{c}{4} \\
\hline Year & 877 & 679 & 586 \\
\hline Stroke & 553 & 422 & 351 \\
Intracerebral infarction & 226 & 175 & 159 \\
Intracerebral hemorrhage & 97 & 81 & 75 \\
Subarachnoid hemorrhage &
\end{tabular}

A

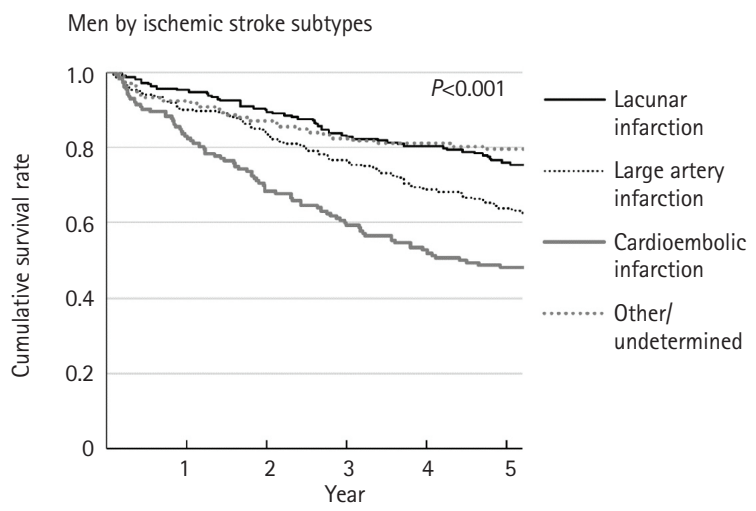

Number at risks

\begin{tabular}{lccr}
\hline Year & 0 & 2 & \multicolumn{1}{c}{4} \\
\hline Lacunar infarction & 208 & 186 & 167 \\
Large artery infarction & 252 & 212 & 173 \\
Cardioem bolic infarction & 152 & 104 & 80 \\
Other/undetermined & 131 & 114 & 106 \\
\hline
\end{tabular}

Women by ischemic stroke subtypes

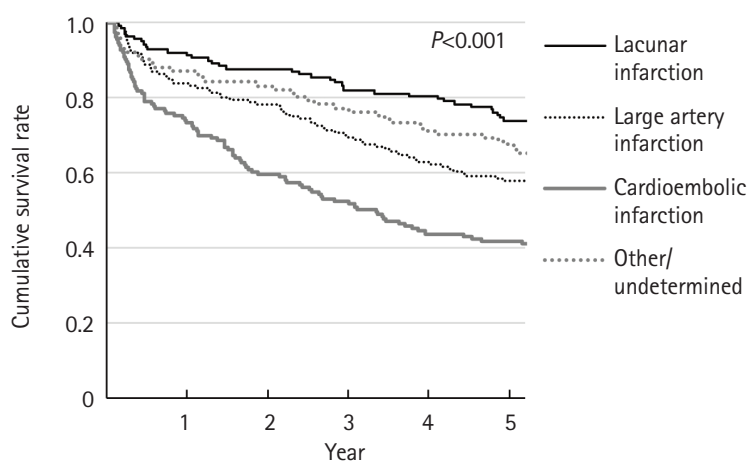

\begin{tabular}{lcrr} 
Number at risks & & & \\
\hline Year & 0 & \multicolumn{1}{c}{2} & \multicolumn{1}{c}{4} \\
\hline Lacunar infarction & 138 & 121 & 111 \\
Large artery infarction & 161 & 126 & 101 \\
Cardioem bolic infarction & 153 & 91 & 67 \\
Other/undetermined & 101 & 84 & 72
\end{tabular}

Figure 1. Five-year survival rate curve after non-fatal first-ever stroke for men by stroke subtypes (A), for men by ischemic stroke subtypes (B), for women by stroke types (C), and for women by ischemic stroke subtypes (D). P-values were calculated by log-rank test across subtypes of stroke (ischemic stroke, intracerebral hemorrhage, and subarachnoid hemorrhage) and ischemic stroke (lacunar infarction, large artery infarction, cardioembolic infarction, and other/undetermined). 
rality rate in the general population using Japanese cohort survival tables. ${ }^{16} \mathrm{~A}$ Cox proportional hazards model was used to evaluate possible predictors for subsequent death. Cases with missing data on TIA or atrial fibrillation $(n=17)$, or a stroke case, not specified as hemorrhage or infarction $(n=1)$ were excluded from the Cox proportional hazards analysis. A $P$-value less than 0.05 was considered statistically significant. All analyses were performed using SAS version 9.4 (SAS Institute, Cary, NC, USA).

\section{Results}

The baseline characteristics of patients after non-fatal first-ever stroke are shown according to type of index stroke (Table 1). A total of 1,880 cases of first-ever non-fatal stroke were ascertained in 2011. The mean patient age was 74.1 years for ischemic stroke and 68.2 years for hemorrhagic stroke. The frequencies of male sex, atrial fibrillation, moderate to severe disability before stroke onset (mRS 3 to 5), and coma (JCS score 100 to 300 ) on admission were $53.4 \%, 17.2 \%, 12.2 \%$, and $7.9 \%$, respectively.

During an average 4.3-year follow-up period, 677 patients died (641 patients died within the 5 years). Table 2 shows the cumulative survival rates after non-fatal first-ever stroke and its subtypes. Among survivors of first-ever stroke, the 5-year cumulative survival rate was $65.9 \%$. The 5 -year cumulative survival rates were $63.5 \%$ for ischemic stroke, $69.1 \%$ for intracerebral hemorrhage, and $77.9 \%$ for subarachnoid hemorrhage. Cumulative survival curves of non-fatal first-ever stroke and ischemic stroke subtypes were plotted separately for men and women (Figure 1).

Table 3. Relative survival rate after non-fatal first-ever stroke by types of stroke and gender in the Shiga Stroke Registry

\begin{tabular}{|c|c|c|c|c|c|c|}
\hline \multirow{2}{*}{ Variable } & \multicolumn{2}{|c|}{ Men } & \multicolumn{2}{|c|}{ Women } & \multicolumn{2}{|c|}{ Men and women } \\
\hline & Survival rate (\%) & $95 \% \mathrm{Cl}$ & Survival rate (\%) & $95 \% \mathrm{Cl}$ & Survival rate (\%) & $95 \% \mathrm{Cl}$ \\
\hline Total stroke & 83.2 & 79.7-86.7 & 80.6 & $76.5-84.6$ & 82.0 & $79.4-84.7$ \\
\hline Ischemic stroke & 83.3 & $79.0-87.5$ & 79.1 & 73.7-84.6 & 81.6 & $78.2-84.9$ \\
\hline Lacunar infarction & 92.1 & $85.1-99.2$ & 93.3 & $84.0-100.0$ & 92.6 & $87.0-98.2$ \\
\hline Large artery infarction & 79.8 & $72.4-87.2$ & 77.9 & $67.7-88.1$ & 79.1 & $73.1-85.1$ \\
\hline Cardioembolic infarction & 65.5 & $54.7-76.4$ & 62.3 & $50.7-73.8$ & 64.0 & $56.1-71.9$ \\
\hline Other/undetermined* & 93.7 & $85.5-100.0$ & 83.2 & 71.9-94.6 & 89.3 & $82.5-96.0$ \\
\hline Hemorrhagic stroke & 83.1 & $76.9-89.3$ & 82.7 & 76.8-88.6 & 82.9 & $78.6-87.1$ \\
\hline Intracerebral hemorrhage & 82.7 & $75.8-89.6$ & 81.9 & $74.5-89.4$ & 82.3 & $77.2-87.4$ \\
\hline Subarachnoid hemorrhage & 85.2 & $71.6-98.8$ & 84.2 & $75.1-93.3$ & 84.5 & $76.9-92.1$ \\
\hline
\end{tabular}

Relative survival rates were calculated the ratio of observational survival rates to the expected survival rates in the age, sex, and calendar year matched general Japanese population. The expected survival rate were calculated by Ederer I methods. ${ }^{15}$

$\mathrm{Cl}$, confidence interval.

*Ischemic stroke with other determined etiology or ischemic stroke with undetermined etiology.

Table 4. Cumulative 5-year survival rate after both nonfatal and fatal first-ever stroke by types of stroke and gender in the Shiga Stroke Registry

\begin{tabular}{|c|c|c|c|c|c|c|c|c|c|}
\hline \multirow[b]{2}{*}{ Variable } & \multicolumn{3}{|c|}{ Men } & \multicolumn{3}{|c|}{ Women } & \multicolumn{3}{|c|}{ Men and women } \\
\hline & $\begin{array}{c}\text { Death/total } \\
\text { no. }\end{array}$ & $\begin{array}{c}\text { Survival rate } \\
(\%)\end{array}$ & $95 \% \mathrm{Cl}$ & $\begin{array}{c}\text { Death/total } \\
\text { no. }\end{array}$ & $\begin{array}{c}\text { Survival rate } \\
(\%)\end{array}$ & $95 \% \mathrm{Cl}$ & $\begin{array}{c}\text { Death/total } \\
\text { no. }\end{array}$ & $\begin{array}{c}\text { Survival rate } \\
(\%)\end{array}$ & $95 \% \mathrm{Cl}$ \\
\hline Total stroke & $454 / 1,138$ & 60.1 & $58.7-61.6$ & $483 / 1,038$ & 53.5 & $51.9-55.0$ & $937 / 2,176$ & 56.9 & $55.9-58.0$ \\
\hline Ischemic stroke & $287 / 783$ & 63.4 & $61.6-65.1$ & $288 / 615$ & 53.2 & $51.2-55.2$ & $575 / 1,398$ & 58.9 & $57.6-60.2$ \\
\hline Lacunar infarction & $50 / 208$ & 76.0 & $73.0-78.9$ & $42 / 144$ & 70.8 & $67.0-74.6$ & $92 / 352$ & 73.9 & $71.5-76.2$ \\
\hline Large artery infarction & $101 / 262$ & 61.5 & $58.4-64.5$ & $84 / 177$ & 52.5 & $48.8-56.3$ & $185 / 439$ & 57.9 & $55.5-60.2$ \\
\hline Cardioembolic infarction & $97 / 170$ & 42.9 & $39.1-46.7$ & $120 / 184$ & 34.8 & $31.3-38.3$ & $217 / 354$ & 38.7 & $36.1-41.3$ \\
\hline Other/undetermined ${ }^{*}$ & $39 / 143$ & 72.7 & $69.0-76.5$ & $42 / 110$ & 61.8 & $57.2-66.5$ & $81 / 253$ & 68.0 & $65.1-70.9$ \\
\hline Hemorrhagic stroke & $153 / 341$ & 55.1 & $52.4-57.8$ & $184 / 411$ & 55.2 & $52.8-57.7$ & $337 / 752$ & 55.2 & $53.4-57.0$ \\
\hline Intracerebral hemorrhage & $123 / 280$ & 56.1 & $53.1-59.0$ & $119 / 271$ & 56.1 & $53.1-59.1$ & $242 / 551$ & 56.1 & $54.0-58.2$ \\
\hline Subarachnoid hemorrhage & $30 / 61$ & 50.8 & $44.4-57.2$ & $65 / 140$ & 53.6 & $49.4-57.8$ & $95 / 201$ & 52.7 & $49.2-56.3$ \\
\hline
\end{tabular}

$\mathrm{Cl}$, confidence interval.

*Ischemic stroke with other determined etiology or ischemic stroke with undetermined etiology. 
Table 5. Multivariable analysis of prognostic predictors for fatality after non-fatal first-ever stroke

\begin{tabular}{|c|c|c|c|c|c|c|}
\hline \multirow{2}{*}{ Variable } & \multicolumn{3}{|c|}{ Hemorrhagic stroke } & \multicolumn{3}{|c|}{ Ischemic stroke } \\
\hline & $\mathrm{HR}$ & $95 \% \mathrm{Cl}$ & $P$ & $H R$ & $95 \% \mathrm{Cl}$ & $P$ \\
\hline \multicolumn{7}{|l|}{ Age (yr) } \\
\hline$<45$ & Reference & & & Reference & & \\
\hline $45-64$ & 6.08 & $0.75-49.45$ & 0.092 & 3.86 & $0.60-24.69$ & 0.153 \\
\hline $65-74$ & 12.13 & $1.49-98.98$ & 0.020 & 7.54 & $1.21-46.79$ & 0.030 \\
\hline $75-84$ & 29.18 & $3.69-230.51$ & 0.001 & 15.67 & $2.53-96.86$ & 0.003 \\
\hline$\geq 85$ & 57.69 & $7.04-472.40$ & $<0.001$ & 37.03 & $5.96-230.24$ & $<0.001$ \\
\hline Women (vs. men) & 0.48 & $0.32-0.72$ & $<0.001$ & 0.59 & $0.47-0.74$ & $<0.001$ \\
\hline \multicolumn{7}{|l|}{ JCS } \\
\hline 0 & Reference & & & Reference & & \\
\hline 1 & 1.77 & $0.97-3.23$ & 0.063 & 1.90 & $1.43-2.52$ & $<0.001$ \\
\hline 2 & 2.65 & $1.37-5.12$ & 0.004 & 1.65 & $1.17-2.33$ & 0.005 \\
\hline 3 & 2.15 & $1.23-3.75$ & 0.007 & 1.72 & $1.26-2.35$ & 0.001 \\
\hline 10 & 1.88 & $1.12-3.16$ & 0.017 & 2.54 & $1.74-3.70$ & $<0.001$ \\
\hline 20 & 4.04 & $1.56-10.44$ & 0.004 & 2.90 & $1.74-4.85$ & $<0.001$ \\
\hline 30 & 2.99 & $1.26-7.12$ & 0.013 & 3.35 & $1.63-6.89$ & 0.001 \\
\hline 100 & 2.16 & $0.87-5.36$ & 0.097 & 3.95 & $2.26-6.91$ & $<0.001$ \\
\hline 200 & 5.26 & $2.86-9.67$ & $<0.001$ & 5.74 & $3.51-9.40$ & $<0.001$ \\
\hline 300 & 10.50 & $5.17-21.33$ & $<0.001$ & 3.36 & $1.60-7.06$ & 0.001 \\
\hline Unknown & 11.94 & $0.89-159.70$ & 0.061 & 6.30 & $3.34-11.90$ & $<0.001$ \\
\hline \multicolumn{7}{|c|}{ History of TIA (vs. no) } \\
\hline Yes & 0.97 & $0.33-2.86$ & 0.952 & 0.88 & $0.59-1.32$ & 0.534 \\
\hline \multicolumn{7}{|c|}{ Hypertension medication (vs. no) } \\
\hline Yes & 0.91 & $0.65-1.26$ & 0.570 & 0.94 & $0.78-1.14$ & 0.539 \\
\hline Unknown & 0.65 & $0.10-4.27$ & 0.652 & 5.78 & $1.22-27.43$ & 0.027 \\
\hline \multicolumn{7}{|l|}{ Diabetes (vs. no) } \\
\hline Yes & 1.33 & $0.90-1.98$ & 0.151 & 1.22 & $0.98-1.50$ & 0.070 \\
\hline Unknown & 1.18 & $0.42-3.34$ & 0.751 & 0.56 & $0.13-2.41$ & 0.436 \\
\hline \multicolumn{7}{|c|}{ Dyslipidemia (vs. no) } \\
\hline Yes & 0.85 & $0.58-1.24$ & 0.388 & 0.79 & $0.65-0.97$ & 0.022 \\
\hline Unknown & 1.11 & $0.61-2.00$ & 0.739 & 1.10 & $0.73-1.67$ & 0.643 \\
\hline \multicolumn{7}{|c|}{ Atrial fibrillation (vs. no) } \\
\hline Yes & 1.58 & $0.95-2.61$ & 0.078 & 1.40 & $1.14-1.73$ & 0.002 \\
\hline \multicolumn{7}{|c|}{ Myocardial infarction (vs. no) } \\
\hline Yes & 1.43 & $0.71-2.89$ & 0.315 & 0.93 & $0.66-1.31$ & 0.664 \\
\hline Unknown & - & - & - & - & - & - \\
\hline \multicolumn{7}{|c|}{ Smoking (vs. nonsmoker) } \\
\hline Past smoker & 0.59 & $0.31-1.12$ & 0.107 & 1.23 & $0.90-1.67$ & 0.196 \\
\hline Current smoker & 1.27 & $0.81-2.01$ & 0.302 & 1.07 & $0.81-1.42$ & 0.619 \\
\hline Unknown & 0.87 & $0.38-1.97$ & 0.730 & 0.66 & $0.43-1.03$ & 0.066 \\
\hline \multicolumn{7}{|c|}{ Drinker (vs. nondrinker) } \\
\hline Past drinker & 3.09 & $1.23-7.76$ & 0.016 & 0.74 & $0.26-2.09$ & 0.569 \\
\hline Current drinker & 0.68 & $0.46-1.02$ & 0.064 & 0.80 & $0.63-1.02$ & 0.072 \\
\hline Unknown & 1.37 & $0.65-2.86$ & 0.411 & 1.25 & $0.90-1.72$ & 0.179 \\
\hline
\end{tabular}


Table 5. Continued

\begin{tabular}{|c|c|c|c|c|c|c|}
\hline \multirow{2}{*}{ Variable } & \multicolumn{3}{|c|}{ Hemorrhagic stroke } & \multicolumn{3}{|c|}{ Ischemic stroke } \\
\hline & $\mathrm{HR}$ & $95 \% \mathrm{Cl}$ & $P$ & $\mathrm{HR}$ & $95 \% \mathrm{Cl}$ & $P$ \\
\hline \multicolumn{7}{|c|}{ mRS before onset } \\
\hline 0 & Reference & & & Reference & & \\
\hline 1 & 1.42 & $0.89-2.27$ & 0.143 & 1.19 & $0.89-1.60$ & 0.243 \\
\hline 2 & 1.62 & $0.90-2.91$ & 0.108 & 1.91 & $1.32-2.76$ & $<0.001$ \\
\hline 3 & 1.45 & $0.71-2.98$ & 0.311 & 1.53 & $1.09-2.15$ & 0.014 \\
\hline 4 & 1.07 & $0.51-2.23$ & 0.863 & 2.37 & $1.71-3.29$ & $<0.001$ \\
\hline 5 & 9.52 & $3.35-27.07$ & $<0.001$ & 2.88 & $1.67-4.97$ & $<0.001$ \\
\hline Unknown & 3.81 & $2.01-7.23$ & $<0.001$ & 2.24 & $0.78-6.44$ & 0.136 \\
\hline
\end{tabular}

HR, hazard ration; $\mathrm{Cl}$, confidence interval; JCS, Japan Coma Scale (JCS 0, alert; JCS 1-3, possible eye-opening, not lucid; JCS 10-30, possible eye-opening upon stimulation; JCS 100-300, no eye-opening and coma); TIA, transient ischemic attack; mRS, modified Rankin Scale.

Table 3 shows the relative survival rates of non-fatal first-ever stroke and its subtypes. The 5-year relative survival rate after non-fatal first-ever stroke was $82.0 \%$. The 5 -year relative survival rates were $81.6 \%$ for ischemic stroke (lacunar infarction, 92.6\%; large-artery infarction, 79.1\%; and cardioembolic infarction, 64.0\%), 82.3\% for intracerebral hemorrhage, and 84.5\% for subarachnoid hemorrhage. The 5 -year excess mortality risk after non-fatal stroke was 1.75-fold higher than that among the general Japanese population. Excess mortality risks were 1.66 for ischemic stroke (lacunar infarction, 1.32; large-artery infarction, 1.75; and cardioembolic infarction, 1.88), 1.93 for intracerebral hemorrhage, and 2.84 for subarachnoid hemorrhage.

As for sensitivity analysis, the 5-year cumulative survival rates after both fatal and non-fatal first-ever stroke are shown in Table 4. Approximately 30\% (296 out of 937) of fatal cases were observed during the first 28 days after stroke onset. The 5 -year cumulative survival rate after first-ever stroke was $56.9 \%$. Five-year cumulative survival rates were $58.9 \%$ for ischemic stroke, $56.1 \%$ for intracerebral hemorrhage, and $52.7 \%$ for subarachnoid hemorrhage. The 5-year relative survival rates after both fatal and non-fatal first-ever stroke were $76.6 \%$ for ischemic stroke, $68.6 \%$ for intracerebral hemorrhage, and $58.7 \%$ for subarachnoid hemorrhage.

Results of multivariable analysis for predictors of death after non-fatal first-ever stroke are shown in Table 5. Age, male sex, higher JCS score, atrial fibrillation, and disability before stroke onset were associated with a higher risk of subsequent death after non-fatal first-ever ischemic stroke; dyslipidemia was associated with a lower risk of future death. In non-fatal first-ever hemorrhagic stroke, age, male sex, JCS score, past drinker, and disability before onset were associated with subsequent death.

\section{Discussion}

In the present study using data of a large-scale, recent comprehensive population-based stroke registry, we demonstrated the current status of 5-year prognosis after first-ever stroke in a real-world setting of Japan. The 5-year cumulative survival rate after non-fatal first-ever stroke was 65.9\%. Lower 5-year cumulative survival rates were observed for cardioembolic infarction (44.9\%) and large-artery infarction (61.5\%). The relative 5-year survival rate after non-fatal first-ever stroke was $82.0 \%$, and lower relative survival rates were observed for cardioembolic infarction (64.0\%) and large-artery infarction (79.1\%). Older age, male sex, higher JCS score on admission, and higher mRS score before stroke onset were associated with higher risk of subsequent death in both non-fatal first-ever ischemic and hemorrhagic stroke.

Five-year cumulative survival rates have been reported to be $50 \%$ to $70 \%$ after total stroke $\mathrm{e}^{6-8,17,18}$ and $30 \%$ to $40 \%$ after intracerebral hemorrhage ${ }^{19-21}$ in studies conducted during the 1980s, 1990s, and early 2000s. In the present study conducted during the 2010s in Japan, whose participants were slightly older than those of many previous studies, we demonstrated similar 5-year survival rates in a sensitivity analysis using 2,176 fatal/no-fatal first-ever stroke patients. These findings suggest possible improvement in age-standardized 5-year survival rates during the past few decades, which might be derived from improvement in stroke care, advances in medical technology, and/ or reduction of stroke severity owing to improved management of cardiovascular risk factors, such as hypertension.

Cumulative survival rates might not be a useful indicator for older patients whose risk of death is very high. In our study, therefore, we also used the relative survival rate, which is an established method in population-based cancer epidemiology $\mathrm{y}^{22}$ for examining long-term survival. Relative survival rate is a 
method to evaluate a corrected survival rate without cause of death information. To our knowledge, this is the first report to calculate recent relative survival rates after non-fatal stroke over the 5-year study period. We also estimated the excess mortality risk after non-fatal stroke over the 5 -year study period to be approximately 1.8 -fold. These findings are consistent with prior studies reporting the excess mortality risk after nonfatal stroke for more than 5 years as 1.9- to 2.9-fold greater. ${ }^{6-8}$

In this study, older age, male sex, severity of index stroke, and disability (mRS) before onset of the index stroke increased the risk of future death after non-fatal first-ever stroke. These findings are consistent with prior review articles of intracerebral hemorrhage, which reported old age and severity of the index stroke as predictors for long term fatality, ${ }^{20,23}$ as well as epidemiological studies reporting that the pre-stroke Barthel Index was associated with subsequent death after non-fatal first-ever stroke. ${ }^{6}$ Cumulative and relative survival rates in men were better than these in women. The discrepancy might be due to that mean age of stroke onset in women was 6 years older than that in men ( 75 years vs. 69 years). We previously reported that high JCS scores on admission predicted fatality at more than 2 years after stroke ${ }^{10}$ as well as short-term death. ${ }^{24,25}$ In this study, we confirmed these prior findings and demonstrated that the association between high JCS scores on admission and high case fatality persists for more than 5 years after first-ever non-fatal ischemic and hemorrhagic stroke.

To our knowledge, this is the first study to report the current status of 5-year prognosis for type-specific stroke based on a large-scale, comprehensive, population-based registry in Asia. This study also has several limitations. First, owing to a lack of information about the causes of death, we could not examine cause-specific survival rates. Second, we only used information obtained from death certificates for residents of Shiga Prefecture. Patients with stroke who relocated after stroke onset were lost to follow-up. Therefore, the survival rates after stroke onset might have been overestimated. However, according to reports on internal migration derived from the Basic Resident Registers, 5.1\% of residents moved out of Shiga Prefecture during the 5-year study period.

\section{Conclusions}

In the present study using data of a large-scale comprehensive population-based stroke registry in a real-world setting of Japan, resent 5-year survival after non-fatal first-ever stroke remained low, particularly after cardioembolic infarction and large-artery infarction. These findings reemphasize the importance of secondary stroke prevention in Japan.

\section{Supplementary materials}

Supplementary materials related to this article can be found online at https://doi.org/10.5853/jos.2020.00325.

\section{Disclosure}

The authors have no financial conflicts of interest.

\section{Acknowledgments}

We thank all investigators, participating hospitals, and study staff at the Shiga Stroke Data Center, Shiga Medical Association, Shiga Prefecture Hospital Association, Biwako Brain \& Heart Attack Consortium (BIWA-BHAC), Department of Public Health Care and Welfare, and the Shiga Prefectural government. We thank Analisa Avila, ELS, of Edanz Group (www.edanzediting.com/ac) for language polishing a draft of this manuscript.

This work was supported by Shiga Prefecture, Japan and the Japan Agency for Medical Research and Development (grant number 17ek0210090).

\section{References}

1. Ueshima H. Explanation for the Japanese paradox: prevention of increase in coronary heart disease and reduction in stroke. J Atheroscler Thromb 2007;14:278-286.

2. Results of vital statistics, 2017. Ministry of Health, Labour and Welfare. https://www.e-stat.go.jp/SG1/estat/GL08020101.do? toGL08020101_\&tstatCode $=000001028897 \&$ requestSender= dsearch. Accessed August 17, 2020.

3. The results of Comprehensive Survey of Living Conditions, 2016. Ministry of Health, Labour and Welfare. https://www. mhlw.go.jp/toukei/saikin/hw/k-tyosa/k-tyosa16/index.html. Accessed August 17, 2020.

4. GBD 2016 Stroke Collaborators. Global, regional, and national burden of stroke, 1990-2016: a systematic analysis for the Global Burden of Disease Study 2016. Lancet Neurol 2019;18: 439-458.

5. Takashima N, Arima H, Kita Y, Fujii T, Miyamatsu N, Komori $M_{1}$ et al. Incidence, management and short-term outcome of stroke in a general population of 1.4 million Japanese: Shiga Stroke Registry. Circ J 2017;81:1636-1646.

6. Hankey GJ, Jamrozik K, Broadhurst RJ, Forbes S, Burvill PW, Anderson CS, et al. Five-year survival after first-ever stroke and related prognostic factors in the Perth Community Stroke Study. Stroke 2000;31:2080-2086.

7. Brønnum-Hansen $H$, Davidsen $M$, Thorvaldsen $P$; Danish 
MONICA Study Group. Long-term survival and causes of death after stroke. Stroke 2001;32:2131-2136.

8. Kiyohara Y, Kubo M, Kato I, Tanizaki Y, Tanaka K, Okubo K, et al. Ten-year prognosis of stroke and risk factors for death in a Japanese community: the Hisayama study. Stroke 2003;34:23432347.

9. Annual report on the ageing society FY 2019. Cabinet Office. https://www8.cao.go.jp/kourei/whitepaper/w-2019/html/ zenbun/index.html. Accessed August 17, 2020.

10. Takashima N, Arima H, Kita Y, Fujii T, Miyamatsu N, Komori $M$, et al. Two-year survival after first-ever stroke in a general population of 1.4 million Japanese: Shiga Stroke Registry. Circ J 2018;82:2549-2556.

11. Tunstall-Pedoe H. Monitoring trends in cardiovascular disease and risk factors: the WHO "Monica" project. WHO Chron 1985;39:3-5.

12. Adams HP Jr, Bendixen BH, Kappelle ப, Biller J, Love BB, Gordon $D L$, et al. Classification of subtype of acute ischemic stroke. Definitions for use in a multicenter clinical trial. TOAST. Trial of Org 10172 in Acute Stroke Treatment. Stroke 1993;24:35-41.

13. van Swieten JC, Koudstaal PJ, Visser MC, Schouten HJ, van Gijn J. Interobserver agreement for the assessment of handicap in stroke patients. Stroke 1988;19:604-607.

14. Ohta T, Waga S, Handa W, Saito I, Takeuchi K. New grading of level of disordered consiousness. No Shinkei Geka 1974;2:623627.

15. Estève J, Benhamou $E_{1}$ Raymond L. Statistical methods in cancer research. Volume IV. Descriptive epidemiology. IARC Sci Publ 1994;128:1-302.

16. Japanese cohort survival table. Center for Cancer Control and Information Services, National Cancer Center Japan. Available at: http://ganjoho.jp/reg_stat/statistics/qa_words/ cohort01.html. 2020. Accessed August 17, 2020.
17. Hardie K, Hankey GJ, Jamrozik K, Broadhurst RJ, Anderson C. Ten-year survival after first-ever stroke in the perth community stroke study. Stroke 2003;34:1842-1846.

18. Imai $A$, Suzuki $H$, Watanabe $T$, Umeyama $N$, Tsukada M, Nakamura $T$, et al. An observational study of stroke patients on the prognosis and causes of death in 5 years: comparison of the results in Tochigi prefecture with the reports in the U.S.A. Jpn J Stroke 2010;32:572-578.

19. Hansen BM, Nilsson OG, Anderson H, Norrving B, Säveland $H$, Lindgren A. Long term (13 years) prognosis after primary intracerebral haemorrhage: a prospective population based study of long term mortality, prognostic factors and causes of death. J Neurol Neurosurg Psychiatry 2013;84:1150-1155.

20. Poon MT, Fonville AF, AI-Shahi Salman R. Long-term prognosis after intracerebral haemorrhage: systematic review and meta-analysis. J Neurol Neurosurg Psychiatry 2014;85:660667.

21. Fogelholm R, Murros K, Rissanen A, Avikainen S. Long term survival after primary intracerebral haemorrhage: a retrospective population based study. J Neurol Neurosurg Psychiatry 2005;76:1534-1538.

22. Compton CC. AJCC Cancer Staging Atlas. 7 ed. New York: Springer-Verlag, 2012.

23. Pinho J, Costa AS, Araújo JM, Amorim JM, Ferreira C. Intracerebral hemorrhage outcome: a comprehensive update. J Neurol Sci 2019;398:54-66.

24. Lee J, Morishima T, Kunisawa S, Sasaki N, Otsubo T, Ikai H, et al. Derivation and validation of in-hospital mortality prediction models in ischaemic stroke patients using administrative data. Cerebrovasc Dis 2013;35:73-80.

25. Shigematsu $K$, Nakano $H$, Watanabe $Y$. The eye response test alone is sufficient to predict stroke outcome: reintroduction of Japan Coma Scale: a cohort study. BMJ Open 2013;3:e002736. 
Supplementary Table 1. Description of Japan Coma Scale $e^{14}$

\begin{tabular}{ll}
\hline JCS levels & \\
\hline 0 & Alert \\
1 & Almost alert \\
2 & Disoriented \\
3 & Does not recall name and birthday \\
10 & Opens eyes in response to normal voice \\
20 & Opens eyes in response to loud voice or shaking body \\
30 & Opens eyes in response to painful stimuli with repeated call \\
100 & Does not open eyes but respond with movements to avoid painful stimuli \\
200 & Does not open eyes but respond with slight movements to avoid painful stimuli (including decerebrate and decorticate response) \\
300 & Does not open eyes and make any movements in response to painful stimuli \\
\hline
\end{tabular}

JCS, Japan Coma Scale. 